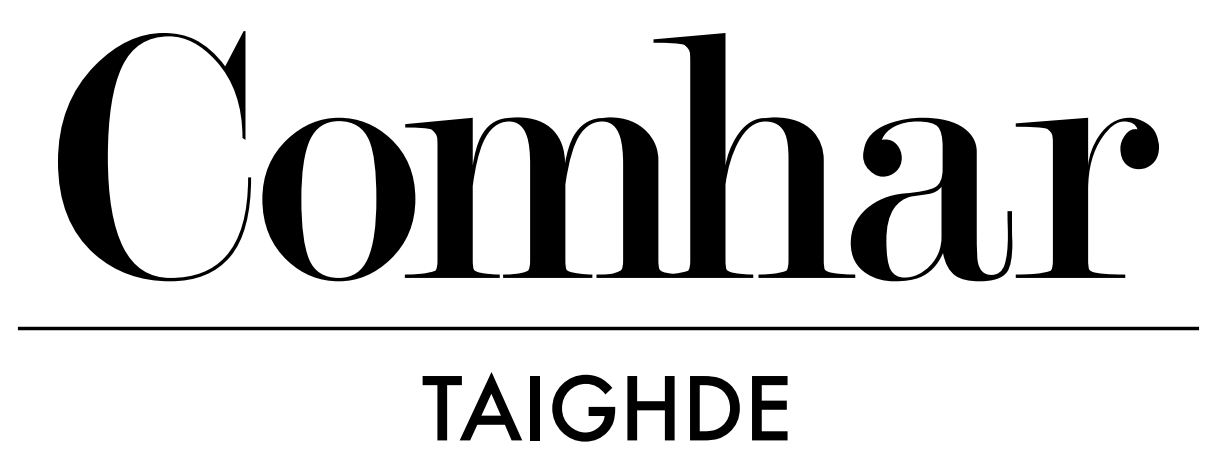

EAGRÁN 4

Samhain 2018

Alt Taighde

'Ag imeacht le sruth an tsaoil': súil eile ar ghnéithe den litríocht taistil

Dáta foilsithe:

30 Samhain 2018

Cóipcheart:

(C) Pádraig de Paor, 2018

Seoladh gréasáin:

http://comhartaighde.com/eagrain/4/depaor/
Údar:

Pádraig de Paor

Comhfhreagras:

pdepaor@tcd.ie

Seoladh seasmhach (DOI):

https://doi.org/10.18669/ct.2018.01

Arna fhoilsiú ag COMHAR Teoranta le cabhair deontais i gcomhair tograí Gaeilge a d'íoc an tÚdarás um Ard-Oideachas trí Choláiste na hOllscoile, Corcaigh. Gabhann COMHAR buíochas le hAcadamh Ríoga na hÉireann as a dtacaíocht leis an bhfiontar seo.

\title{
comhartaighde.ie
}

ISSN: 2009-8626 


\section{'Ag imeacht le sruth an tsaoil': súil eile ar ghnéithe den litríocht taistil}

\section{Pádraig de Paor}

\section{Achoimre}

Scrúdaíonn an t-alt seo gnéithe den litríocht taistil sa Ghaeilge. Áitítear nach mór idirdhealú a dhéanamh idir an scríbhneoireacht taistil nach bhfuil inti ach cuntas agus an litríocht taistil ar saothar ealaíonta de chuid na samhlaíochta í. Féachtar le modh eile léitheoireachta a fhorbairt nach leanann téarmaí tagartha na ndioscúrsaí atá thuas i ngort sin na critice ar an litríocht taistil sa Ghaeilge faoi láthair (an chéannacht agus an eileacht, idirchultúrachas, inscne, polaitíocht teanga). Ina ionad sin, féachtar le modh léitheoireachta a fhorbairt a chuireann pléisiúr an taistil agus pléisiúr na léitheoireachta ag croí an scrúdaithe ar an litríocht taistil. Chuige sin, baintear leas as trí choincheap: An dul le sruth nó 'flow', an carnabhalachas nó 'carnvalesque' agus an t-eispéireas teorann nó 'limit experience'.

Tá litríocht taistil á foilsiú go tréan sa Ghaeilge le scór éigin bliain. Ach cad atá i gceist leis an 'litríocht taistil'? Litríocht a éiríonn as turas a thugtar ar thír éigin thar lear, is dócha. In amanna, déantar idirdhealú idir litríocht na deoraíochta agus an litríocht taistil, idir an litríocht a spreagann an imirce agus í sin a scríobhtar faoin turas a roghnaítear, nó a mbaintear pléisiúr as (Ní Dhonnchadha agus Nic Eoin 2008: 17-18). D'fhéadfaí an t-idirdhealú sin a cheistiú. Thig le heisimirceach a mhaíomh gur cuid dhosheachanta den saol é go gcaithfidh sé imeacht ar imirce nuair is fonn taiscéalaíochta, fonn éalaithe nó tóir ar shaoirse phearsanta is bun leis an imeacht i ndáiríre. Ar an láimh eile, is iomaí turas pléisiúrtha nó saoire thar lear atá ceangailte leis an obair. Nuair a fuair Liam Ó Rinn mí 'laethanta saoire' i Meán Fómhair 1924, thug sé turas traenach go Páras air féin, ach níor éirigh leis an obair a fhágáil taobh thiar de go hiomlán. Mórán gach aon lá le linn na míosa sin, sheoladh sé cuntas ar a eachtraí chuig The Freeman's Journal. Tá na haistí sin bailithe le chéile sa leabhar Turus go Páras (1931). Tugann Séamus Ó Grianna cuntas in Saol Corrach (1945) ar an leathbhliain a chaith sé i ndeisceart na Fraince in 1926-7 ag fáil bhisigh ó aicíd scamhóg. B'éigean dó post a ghlacadh thall ag teagasc Béarla chun é féin a choinneáil. Tamall maith de bhlianta ina dhiaidh sin, phill sé chun na Fraince lena bhean ar a slí chun na Róimhe ar oilithreacht. Cé go dtig léi a bheith pléisiúrtha, is 'obair' nó turas dualgais í an oilithreacht ó bhun, turas a mbíonn a sciar féin den dua ag baint léi. Idir 1953 agus 1956, bhíodh an colún 'Caiscín' in The Irish Times ag Máirtín Ó Cadhain, mar ar scríobh sé faoina chuid taistil in Albain agus sa Spáinn (Ó Cathasaigh 1998). Arís, cé nach don obair go sonrach a rinne sé na turasanna sin, ní raibh siad gan cheangal lena chuid oibre ach oiread. Choinnigh 
Micheál Mac Liammóir 'dialann mheánmhara' fad is bhí sé ag taisteal mar chuid dá ghairm aisteoireachta lena chompántas drámaíochta san Éigipt agus i Malta (Mac Liammóir 1956). Scríobh Úna Ní Mhaoileoin dhá leabhar taistil faoin Iodáil ag éirí as turas staidéir agus as a gairm mar ealaíontóir, agus leabhar eile faoi shaoire taighde a chaith sí sa Tuinéis. (Ní Mhaoileoin 1958, 1967, 1969). Luaitear an beagán samplaí sin ar dhá chúis, i) chun a léiriú go dtéann an seánra siar roimh an ré chomhaimseartha, agus ii) nach rud annamh é an tsaoire taistil sin a bheith ceangailte le saothar agus le slí bheatha an údair agus go mb'fhéidir deoraíocht shealadach a thabhairt ar an turasóireacht sin go fiú. Ní foláir go mbaineann cuid den bhláthú atá faoin litríocht taistil le glúin anuas, ní hamháin le borradh sa gheilleagar, ach fosta le borradh sna slite beatha sin a cheadaíonn an taisteal nó a éascaíonn an taisteal, agus go deimhin, slite beatha a gcothaíonn an taisteal iad.Is éTG4 an sampla is fearr, b’fhéidir. Rinne Dermot Somers, Cathal Ó Searcaigh agus Manchán Magan cláracha teilifíse faoin taisteal do TG4 agus scríobh siad leabhair faoina gcuid taistil. Is ag taisteal mar chuid dá dhualgas airm a bhí Frank Reidy, i dtosach. Mar sin de, tá an gaol suimiúil sin idir an tsaoire agus an saothrú ina ghné den litríocht taistil a ndéantar dearmad de, seans, san idirdhealú ródhocht sin idir litríocht na himirce agus an litríocht taistil.

Tá léamh suimiúil déanta cheana féin ar scríbhneoireacht taistil na Gaeilge ag Michael Cronin, ag éirí as a léitheoireacht leathan intleachtúil atá fréamhaithe i léann an aistriúcháin (Cronin 2000). Ina theannta sin, tá an scríbhneoireacht taistil scrúdaithe ag Kate Uí Chíobháin agus Eimear Ní Chinnéide (Uí Chíobháin 2015; Ní Chinnéide 2015). Leagann siad beirt béim ar pheirspictíocht shainiúil shochtheangeolaíoch na dtaistealaithe Gaelacha, ar cainteoirí teanga atá idir mhionlaithe agus á hathréimiú iad. Díol suime, dá réir, dar leo beirt, iarrachtaí na nGael i gcéin a bheith níos tuisceanaí faoi íogaireachtaí cultúrtha ná mar a bheadh an cainteoir Béarla aonteangach, b’fhéidir, go fiú más iarrachtaí útamálacha in amanna iad. Scrúdaíonn Uí Chíobháin agus Ní Chinnéide na slite ina bpléann scríbhneoirí éagsúla taistil le ceist choigilteach na comhbhá agus an chomhionannaithe (Uí Chíobháin 2015: 243-258; Ní Chinnéide 2015: 259-278). Is ag plé le scríbhinní liteartha mar cháipéisí sóisialta, seachas mar litríocht atá siad, áfach.Mar chodarsnacht, san aiste seo, ní hí an 'scríbhneoireacht' taistil is suim liom ach an 'litríocht' taistil, is é sin, scríbhneoireacht taistil ar litríocht í. Ní dhéanfar suirbhé ach tagairt roghnach do roinnt leabhar taistil a chuaigh i bhfeidhm orm agus a bhfuil fiúntas éigin liteartha ag baint leo, dar liom. Ní leantar cur chuige socheolaíoch, tíreolaíoch nó polaitiúil anseo, ná ní bhacfar mórán le téarmaí tagartha polaitiúla na ndioscúrsaí atá thuas sna daonnachtaí faoi láthair (díospóireachtaí faoin chéannacht agus faoin eileacht, faoi ilchultúrthacht, idirchultúrachas, ciníochas, inscne, an spás diaspórach srl). Ina áit sin, is ar ghné eile den litríocht taistil a dhírítear san aiste: an pléisiúr. Bíonn an pléisiúr ag croí an taistil, agus bíonn an pléisiúr ag croí na litríochta agus na léitheoireachta chomh maith. Sa dá chás fosta, bíonn saothar áirithe nach beag go minic ag baint le haimsiú an phléisiúir. Mar sin de, an cheist a spreagann an aiste seo ná: cad é mar a thig linn an litríocht taistil a léamh agus a thuiscint ar shlí a thugann a cheart don phléisiúr sin? Féachtar le léamh a dhéanamh nach mbeadh piúratánach (faoi mar atá an léamh polaitiúil, dar liom).

Chun eispéireas an taistil a scrúdú ar shlí a thugann a ceart do phléisiúr an taistil, leanaim, go scaoilte, Julia Harrison, Being a Tourist: Finding Meaning in Pleasure Travel (1993). Ó pheirspictíocht na hantraipeolaíochta sóisialta de, scrúdaigh sí an bhrí a thugann taistealaithe Ceanadacha dá gcuid eachtraíochta thar lear. Chuir Harrison agallamh ar thaistealaithe tiomanta agus rinne achoimre ar na téamaí is tábhachtaí a thagann aníos sna cainteanna a dhéanann na taistealaithe faoi bhrí a gcuid taistil. Tarraingítear ar ghnéithe áirithe dá taighde san aiste seo ag pointí éagsúla, de réir mar a oireann. Thar rud ar bith, leagfar béim ar an pharadacsa go mbíonn turasóirí iartharacha comhaimseartha ar thóir an phléisiúir, ach fós sásta cur suas le go leor anró agus stró le linn a gcuid eachtraíochta chun 'pléisiúr' sin a taistil a bhaint amach.

Níor mhór a aithint go gcleachtann an léitheoir féin an turasóireacht chomh maith. Mar sin de, bheifí ag súil le toise éigin athfhillteach an fhéinscrúdaithe a bheith in aon léamh a dhéanfaí ar an litríocht taistil. B'éigean don léitheoir seo athbhreithniú a dhéanamh ar an éirí in airde a bhí ag baint lena dhearcadh féin ar an taisteal in amanna: ag samhlú go raibh barántúlacht ag baint le heachtraíocht neamhspleách 
an 'taistealaî'; barántúlacht nach raibh le fáil in aistear eagraithe an 'turasóra', turas treoraithe a d'fhágfadh dall an duine ar mhuintir na háite agus ar a gcultúr. Cuidíonn cur chuige Harrison linn éalú, beagáinín, ón chontúirt sin titim chun na snobaireachta, mar ní éiríonn sí gafa ar fad i ndíospóireachtaí faoi bharántúlacht cineálacha taistil thar a chéile. Mar sin de, cén bhrí a d'fhéadfadh a bheith ag an taisteal, ag an turasóireacht, do na daoine a chleachtann sin sa lá atá inniu ann? Cad é a spreagann agus a ghríosann daoine chun a gcuid ama, a gcuid fuinnimh agus a gcuid airgid a chaitheamh ar an taisteal nach mbíonn i gcónaí éasca nó compordach. Scrúdaíonn Harrison an infheistíocht mhór a dhéanann taistealaithe ina gcuid eachtraíochta: an infheistíocht mhothálach, infheistíocht acmhainní fisiciúla agus síceolaíochta, gan trácht ar infheistíocht airgid. Dar léi, is tóraíocht phearsanta de shaghas atá san eachtraíocht thar sáile (Harrison 1993: 154-163). Tóraíocht í a bhfuil toise quasi-spioradálta ag baint léi, dar liom. Is dóigh liom go gcuidíonn an taisteal leis an duine 'olc' éigin ann féin a phurgú: olc na hoibre nó olc éigin eile a bhíonn ag luí ar an taistealaí.

Cad iad na mianta doimhne a spreagann tóraíocht i gcéin sin an taistealaí? Dar le Harrison (1993) seo thíos cuid acu: i) an tóir ar chaidreamh agus ar nasc le daoine eile; an tóir ar chineál eile caidrimh nach bhfuil ar fáil go furasta sa bhaile; ii) an tóir ar stíl phearsanta, nó ar thoighis phearsanta a fhorbairt; iarracht ar aeistéitic phearsanta a chothú agus a chur in iúl; iii) an tóir ar fhéintuiscint, an tóir ar ionad an duine aonair sa domhan a thuiscint; an tóir ar thuiscint níos doimhne den bhaile agus den dúchas trí 'staonadh' ó shuaimhneas na máithrí seal: Is soiléire an bhrí atá le 'a bheith sa bhaile' nuair a bhítear as baile; agus iv) an tóir ar chiall a dhéanamh de chuid de chastachtaí cultúrtha na cruinne. Le linn an taistil, bíonn seans praiticiúil ag an duine scrúdú a dhéanamh ar chás staidéir beo den tslí a mbuaileann an stair, an pholaitíocht, an tíreolas, an teanga agus an reiligiún le chéile i dtír éigin, i bpobal éigin. Go hachomair, seans atá sa taisteal i gcéin machnamh a dhéanamh ar an saol, machnamh ar bheatha phearsanta an taistealaí agus ar a bheith ann i gcoitinne. Uirlis féintuisceana atá sa litríocht a dhéantar le linn an taistil nó a éiríonn as an taisteal.
Ar a thaisteal dó, bíonn seans in athuair ag an turasóir suim a chur in aer an tsaoil. Cuidíonn éiginnteacht agus oscailteacht na saoire le cothú na sceitimíní, na saoirse agus, thar rud ar bith, le cothú an dlúthchaidrimh. Dar le Harrison arís, is ar thóir caidrimh le strainséirí a bhítear go minic le linn an taistil (Harrison 1993: 43-91). Féach an bhéim a leagann Cathal Ó Searcaigh, mar shampla, ar a chaidreamhúla is atá muintir Kathmandu:

Tá an chathair seo iontach fáilteach, iontach fiosrach, iontach fiafraitheach. Mura n-éiríonn leat caidreamh a dhéanamh ar na sráideanna seo, ort féin atá an locht. Ní dhúnfar doirse an doichill romhat anseo, ach a mhalairt ar fad... osclófar iad le haoibh an gháire. Tá bunadh Neipeal oscailte, fial lena ndáimh. Tá siad cineálta, croíuil, agus is furasta cumann a dhéanamh leo. Tá siad beo bocht. An t-aon rud luachmhar atá acu le tabhairt duit ná a ngáire agus tugann siad sin go buíoch, beannachtach, gan fiacail a chur ann (Ó Searcaigh 2004: 13).

\section{Tairseachúlacht an taistil}

Ó tharla go mbíonn an taistealaí níos leochailí thar lear ná mar a bhíonn sé sa bhaile, tugann an turasóireacht seans dó dul sa seans ar strainséirí, a muinín a chur iontu, agus blas a fháil ar chineál eile dlúthchaidrimh, nach bhfuil fáil réidh air sa ghnáthshaol sa bhaile. Tá rud éigin le déanamh ag an dlúthchaidreamh le hoscailteacht na soghontachta. De thairbhe a leochaileachta i bhfad ó bhaile, bíonn an taistealaí ag brath ar strainséirí. Nuair a thagann strainséir i gcabhair ar an taistealaí, téann an cineáltas sin i bhfeidhm air ar shlí dhomhain nach ndéanfadh sa bhaile. Bíonn dlúithe agus déine leis an chaidreamh i gcomhthéacs sin na leochaileachta. Seans go gcuireann deacrachtaí teanga leis an dlúthchaidreamh go minic seachas a mhalairt; bíonn ar an taistealaí dul i muinín na ngeáitsí, agus an gháire agus na teanga briste chun é féin a chur in iúl. Sa tóir ar chaidreamh le muintir na háite, le treoraithe, le freastalaithe, le tiománaithe tacsaí, le fostaithe a bheas sásta labhairt leis go nádúrtha agus go hionraic a bhíonn Rosenstock in Ólann mo Mbiuiil as an nGainséis (2003). Go fiú más gearrshaolach, dromchlach a bhíonn caidreamh an turasóra leis an dúchasach, ní hionann sin is a rá 
go bhfuil an caidreamh sin mí-ionraic, bréagach nó folamh. Thar aon rud, ba mhaith le Rosenstock a mhothú gur aoi ag an phobal dúchasach é, fiú má aithníonn sé nach cuid d'uige an phobail áitiúil é. Ba mhaith le Rosenstock go mbainfeadh an dúchasach leas nó sult as an idirghabháil fosta. Féach sceitimíní Rosenstock agus é i mbun siopadóireachta san India:

Go dtí seo, d'fhéachas ar an tsiopadóireacht mar dhualgas cuibheasach leamh; ní spreagann earraí tí ná cuirtíní mé. San India, áfach, braitheann tú sceitimíní agus earraí á gceannach agat, dathanna agus patrúin á bhfeiscint agat ná facaís riamh cheana. An bród atá ar lucht a ndíolta, is bród tógálach é. Bíonn bród ort féin gur ball den gcine daonna thú, an cine sin atá in ann earraí chomh healaíonta sin a shamhlú, a dhearadh agus a chruthú. Braitheann tú gur pribhléid ab ea é gur ligeadh isteach sa siopa in aon chor thú. Roinneadh rúneolas leat! Bíonn mórtas ort as an traidisiún, as an lámhcheardaíocht, bíodh is nach iad do mhuintir féin a dhein iad. Braitheann tú umhal, agus buíoch! (Rosenstock 2003: 56).

As baile, mothaíonn an taistealaí stoite óna mháithreach agus, dá réir, níos tuisceanaí ar cé na daoine sa bhaile, agus cé na nithe sa bhaile, atá tábhachtach. Neartaíonn tairseachúlacht an taistil na mothúcháin sin a bhaineann leis an ghrá agus leis an tsearc, ar láimh amháin, agus ar láimh eile neartaíonn tairseachúlacht an taistil an t-uaigneas, ar 'eispéireas teorann' comónta de chuid an taistealaí fhiontraígh é:

Tháinig uaigneas orm agus do bhraitheas ná féadfainn cur suas lena thuille de Pháras dá áilleacht é (Ó Rinn 1931: 95).

Ní raibh bád ná árthach le feiscint in aon áit ná aon solas daonna. Bhí an chriú go léir ina gcodladh is mé i m'aonar ar deic. Bhraitheas tocht beag uaignis (Mac Síthigh 2013: 235).

\section{Chaith mé an Geimhreadh agus an tEarrach i} Menton, agus i dtús a' tSamhraidh bhí biseach maith orm. Bhí rún agam cupla mí eile a chaitheamh ann sa dóigh is go mbeadh aimsir mhaith ann nuair a thiocfainn 'un a' bhaile. Acht bhuail tallann toban mé aon lá amháin agus rinne mé amach imtheacht a' lá thar n-a bhárach.... Bhí mé 'mo shuidhe ar thaoibh a' chnuic, agus gan bhréig gan amhras b'aoibhinn an radharc a bhí ós coinne mo shúl. Cap Martin ar a' taoibh thiar agus Bordigheira, san Eadáil, ar a' taoibh thoir agus camus gorm mara eatorra....

Is áluinn a' radharc é, arsa mise liom féin. Acht níl sé comh háluinn le Rann na Feirste. Agus bhuail cumhaidh mé, mar bhuail Oisín nuair a bhí sé i dTír na hÓige (Ó Grianna 1945: 249).

\section{Eispéireas teorann}

Go paradacsúil, tá an 't-eispéireas teorann' sin (an t-uaigneas, cuirim i gcás) le háireamh mar chuid éigin de tharraingt an taistil, eispéireas foircneach a chuireann brú chomh mór sin ar chorp is ar intinn an duine go gcraitear é go hiomlán as a zón compoird, go hiomlán as a ghnáthstaid mhothachtála is mhachnaimh. Mar seo a leanas a chuireann Charles Taylor síos ar an eispéireas teorann': ‘...] an experience which unsettles and breaks through our ordinary sense of being in the world, with its familiar objects, activities and points of reference [...] when "ordinary reality" is "abolished" and something terrifyingly other shines through' (Taylor 2007: 5). Luadh cheana an paradacsa tábhachtach de chuid an taistil 'phléisiúrtha' nach mbíonn sé i gcónaí chomh pléisiúrtha sin. Teacht slán is ea an taisteal in amanna: eispéireas a fhulaingt, seachas scíste agus sómas. Thig le heispéireas an taistil a bheith an-mhíchompordach agus fiú uafásach. Mar sin féin, le linn an eispéiris teorann sin, le linn an bhrú sin a chuirtear ar an chorp is ar an aigne, tá mar a bheifear ag briseadh tríd chuig réimse eile taithí, réimse taithí atá taobh thall den ghnáthréaltacht. Sa tslí sin, an taistealaí, atá ag fulaingt de bharr an taistil, tuigeann sé an domhan ar mhalairt slí agus tuigeann sé 'a bheith sa domhan' ar shlí eile.

Is fusa dul ag tástáil na dteorainneacha pearsanta thar lear agus gnáthstruchtúr na beatha sa bhaile ar fionraí. Comhartha sóirt de chuid na turasóireachta sa litríocht taistil, is dóigh liom an brú breise a chuirtear ar an chorp, triail an mhíchompoird a chuirtear ar an cholainn. Díríonn cruatan an taistil aird an duine ar a cholainn, ar a chraos agus ar a chuid feidhmeanna nádúrtha: 
I ndiaidh sé huaire ar an bhus seo agus fadharcáin ag teacht as ár dtóin ag na suíocháin chruaidhe mhiotail, ár mboilg thiar ar ár ndroim leis an ocras, mheas muid gurbh fearr stopadh agus an oíche a chaitheamh i sráidbhaile beag Uspantán... Bialann amháin foscailte, seomra beag le tine adhmaid ar ar cócaireáileadh béile leamh go leor os ár gcoinne, ach nárbh é Dia a chuir chugainn é. Leoga, cha raibh mórán de rogha ar bhia-chlár, ach níor chuir aon duine suas don tsú circe agus na tortillas nuadhéanta....

Chaith mé féin mo shúil ar dhoras a raibh fógra scríofa air. 'Sola para orinar' a dúirt an fógra. Thuig mé gur leithreas a bhí ann ceart go leor, agus arsa mise, ag ligint orm go raibh tuigbheáil mhaith agam den Spáinnis: 'Is dóiche go gciallaíonn an fógra sin - do chustaiméirí amháin.' Bhuel, daoine múinte a bhí i mo chomhluadar agus char phléasc siad amach ag gáirí, ach chuir siad ar an eolas mé - ní raibh cead agat an leithreas a úsáid ach de 'ghnaithe' amháin! Níor chuir mé ceist cá háit a rachfá dá mbeadh an dara 'gnaithe' agat. Bhuel, fuair mé dhá fhocal as trí ceart, ar scor ar bith. Cé go raibh seomra agam domh féin i bpension an oíche sin, ní raibh sé en suite agus bhí an leaba cnapánach, callánach agus chuaigh na madaí agus na coiligh ar daoraidh ar fad (Nic Giolla Bhríde 2003: 50, 51).

Leagann cruatan an taistil béim in athuair ar bhunriachtanais chorpartha an duine - cé chomh tábhachtach is atá sé leaba shábháilte a aimsiú don oíche; bia a réiteoidh leis an bholg Éireannach, uisce glan a fháil chun tart a shléachtadh; agus foscadh a aimsiú ó theas na gréine, agus thar rud ar bith eile: leithreas. Ó tharla gur i mbád réasúnta beag a dhéanadh Domhnall Mac Síthigh a iomramh is minic plé tíriúil ina chuntas ar an dúshlán a bhaineann le bia a sholáthar agus riachtanais leithris an taistealaí (Mac Síthigh 2013: 71, 171-3, 273-4).

Thig toise nach mór spioradálta a bheith le heispéireas teorann an taistil: an brú a chuireann an taisteal ar an chorp agus ar an aigne. Fógraíonn Magan go háirithe go bhfuil críoch spioradálta lena shaoire féin, go bhfuil an diantréanas aeiséitiúil ag croí a chuid turasóireachta:
Bhíos tar éis trí mhí a chaitheamh i bhfolach i mbothán cloiche sna Himiléithe gan uisce reatha ná leictreachas. Saol an díthreabhaigh á chleachtadh agam: laethanta cromtha os cionn tine suaraí i mbun machnaimh, nó ag siúl sléibhe ag ithe ríse agus dhal, agus gach maidin gan teip ag ól pionta múin - mo chuid féin gan dabht (Magan 2005: 2).

\section{Imeacht le sruth}

I saothar Magan, go háirithe, faightear samplaí den 'social flying' a chleachtann iartharaigh óga sa tríu domhan dóibh: ag ísliú aicme trí chruatan agus trí shimplíocht na maireachtála agus ag déanamh a mhalairt fosta ó am go chéile: 'd'itheamar féasta an oíche sin lenár bhflúirse scillingí Ugandacha i mbialann ardnósach Shíneach i dteannta oifigigh de chuid na Náisiún Aontaithe a raibh eolas uaidh i dtaobh na Sáíre' (Magan 1998: 114).

Mar sin de, tá praghas ard le híoc ag an taistealaí go fisiciúil agus go síceolaíoch (tuirse, tart, ocras, buinneach, uaigneas, spleáchas ar strainséirí, caiteachas airgid). Ach bíonn rud éigin le gnóthú fosta as eispéireas teorann sin an taistil: an 'dul le sruth' nó an 'flow' (a phléifear anois), agus an carnabhalachas (a phléifear ar ball). 'Flow' a thugann Csikszentmihayi (1992) ar an eispéireas quasi-eacstaiseach sin a thagann i gceist nuair a bhíonn duine gafa go hiomlán le dúshlán a shárú. Fiú an té nach bhfuil aon chur amach aige ar shaothar Csikszentmihayi, tuigfidh sé cad é atá i gceist le bheith 'sa zón': staid intinne áirithe a bhíonn ag duine a bhfuil a aigne go hiomlán dírithe ar ghníomhaíocht thaitneamhach a bhfuil dúshlán ag baint léi. Sáite san iarracht, dearmadann an duine atá ag 'imeacht le sruth' imní agus buairt an tsaoil; cealaítear an t-am agus an spás, de réir dealraimh; braitheann an duine dianfhócasaithe sin go bhfuil an saol go seolta faoina smacht aige. In imeacht le sruth sin na saoire, is tábhachtaí próiseas na gníomhaíochta ná aon toradh a bhíonn air. Iarracht í an tsaoire titim as carcair an amchláir agus na liostaí rudaí atá le cur i gcrích chun titim isteach in eacstais na himeachta le sruth. Nuair a éiríonn leis an taistealaí titim i dtiúin le rithim na háite, bíonn sé ag seoladh le cóir ghaoithe.

'Glac le rithim na háite agus fág i do dhiaidh stró agus brú na linne seo' a mholann Frank Reidy dúinn san Aifric dó (2009: 32): 
Tá rithim mhuintir bhaile Shela fite fuaite leis an mosc, an aimsir agus na séasúir, mar a bhíodh fadó. ... Éiríonn siad leis an lá, fir an bhaile ag déanamh ar an mosc, agus tar éis guí téann siad chomh fada leis an gcéibh. Bíonn na mná ag réiteach gasúr don scoil nó ag cur sna garranta. Feictear na gasúir ina gcuid éide scoile ag spraoi ar a mbealach chuig an scoil náisiúnta atá ar chúl an bhaile. Caitheann na cailíní seál liathdhearg Ioslamach. Níl Críostaithe ar bith sa mbaile seo. Cloistear na hasail ag grágaíl ag iompar lastais aníos ón gcéibh. Rithim nár athraigh leis na cianta, rithim shéasúr na báistí i mbaile Shela (Reidy 2009: 34).

$\mathrm{Ag}$ iarraidh é féin a chur in oiriúint do rithim Kathmandu a bhíonn Ó Searcaigh:

Siúlaim thart sna sráideanna cunga, rúnda seo in Chetrapati, in Thamel agus in Kantipath. Ní léir domh go bhfuil ainmneacha ar bith ar an chuid is mó acu. Anseo is tábhachtaí léargas ná léarscáil. Ar scor ar bith, is fearr liom imeacht liom i mbéal mo chinn ar na sráideanna seo, gan fios agam an siar nó soir atá mo thriall. Lean do ghaosán go réidh, a deirim i dtólamh, agus tiocfaidh do thóin i do dhiaidh. Níl dóigh níos fearr le haithne a chur ar an chathair seo ná do chuid rámhaí a ligean le sruth... imeacht i mbéal na séibe... Ní aimseoidh duine é féin go deo go dtí go mbeidh sé i ndiaidh é féin a chailleadh ar dtús (Ó Searcaigh 2004: 13, 14).

Ag buaic na saoire, siúlann an taistealaíi dtiúin le rithim na ndúchasach sa dóigh is nach dtugann an taistealaí é féin faoi deara: tá sé i gcomaoin leis na dúchasaigh. $\mathrm{Ag}$ buaic sin na saoire, déantar dearmad den am. Ní sheastar siar ag dearcadh ar an saol, ach glactar páirt ann. Cealaítear an dealú idir an ghníomhaíocht agus an meabhrú; ní thógtar grianghraif; ní scríobhtar cuntas. Ach sin í íoróin agus paradacsa de chuid na litríochta taistil: ó tharla go gcailltear eispéireas eacstaiseach sin an 'dul le sruth' nuair a mheabhraítear go féinchomhfhiosach faoi, ní thig le cuntas scríofa ar bith breith i gceart ar bhuaic sin an taistil. Sin an ait a dtagann mianach liteartha an tsaothair i gceist .i. chun cineál eile dul le sruth a chruthú san insint a chuirfeas dul le sruth an taistil in iúl. Ar ndóigh, bíonn dul le sruth ag baint le léitheoireacht na litríochta chomh maith nuair a bhítear sáite i sruth na samhlaíochta agus na scéalaíochta.

\section{An carnabhalachas}

I dteannta na himeachta le sruth, maíonn Julia Harrison go dtig feidhm 'charnabhalach' a bheith leis an turasóireacht. Beidh cur amach ag lucht litríochta cheana ar choincheap an charnabhalachais nó 'the carnavalesque', de réir mar a d'fhorbair Mikhail Bahktin é ina shaothar Rabelais and bis World (1984). Is éard is carnabhalachas ann sa chomhthéacs sin ná stíl. Stíl liteartha í a fhreagraíonn do stíl iompair nó stíl fhéinchurtha-in-iúl. Stíl is ea í atá, ar an dromchla, anordúil, ainrialta, trína chéile, bunoscionn, frithchoinbhinsiúnta. Stíl 'threascrach' í a thugann sos sealadach don té a chleachtann, sos ón ghnáthdhioscúrsa, ón ghnáthstruchtúr agus ó na gnáthrialacha (ach is treascairt cheadaithe 1 atá de réir a chuid coinbhinsiún féin). Cur chuige nó stíl is ea an carnabhalachas liteartha a bhaineann an bonn den stíl choibhinsiúnta údarásach a mbítear ag súil léi i saothar urramach litríochta nó i suíomh measúil ar bith (arís is taobh istigh de pharaiméadair chinnte cheadaithe a thugtar 'fogha' faoin údarás). Tógann Bahktin a choincheap liteartha ar thraidisiúin an charnabhail sna meánaoiseanna, 'féile na n-amadán', cleachtas ina ndéanadh mionchléirigh eaglasta aithris spraíuil ar na searmanais shollúnta a bhíodh ar siúl san ardeaglais. San fhuirseoireacht éadromchroíoch aerach sin, bhristí an tsollúntacht agus dhéanfaí an saol a chasadh bunoscionn go carnabhalach ar feadh tamaillín (Bahktin: 1984: 78-99). Luann Bahktin ceithre thréith le cíor thuathail sin an charnabhalachais: castar daoine le chéile nach gcasfadh le chéile de ghnáth, castar uaisle ar ísle, cuirim i gcás; glactar níos réidhe le haistíl agus le hiompar neamhghnách. Ní bhíonn an brú céanna ar dhaoine a mianta 'nádúrtha' a choinneáil faoi smacht na measúlachta; ceadaítear an treascairt agus an sárú dlí, ar shlí shiombalach, gan aon phionós a bheith le híoc as; bíonn comhghuaillíocht neamhghnách idir páirtithe nach samhlófaí le chéile go coinbhinsiúnta iad, cuirim i gcás, idir óg is aosta. Baintear leas níos leithne as an téarma sa lá atá inniu ann, seachas mar a bhaineadh Bahktin. Mar sin de, sa chiall leathnaithe sin, nach bhféadfaí leas as an charnabhalachas chun an 
cineál 'mí-iompair' a chleachtann lucht turasóireachta in amanna a thuiscint?

Éiríonn leis an taistealaí, atá i ndiaidh imeacht go mór amach óna zón compoird, breith ar chuid éigin de spiorad sin an charnabhalachais ina chuid eachtraí i dtír iontais atá bunoscionn lena bhfuil taithí aige air. Téarma défhiúsach é an carnabhalachas, agus sílim go bhfuil sé an-chabhraíoch chun iompar an taistealaí a thuiscint. Cuidíonn sé sin linn plé le pléisúr na turasóireachta agus lena litríocht, i dtéarmaí nach bhfuil diúltach uilig i gcónaí, cuirim i gcás, an 'héadónachas'. Ní gá, tar éis an tsaoil, gur sa tóir ar an phléisiúr amháin a bheadh an 'héadónaî', ach sa tóir ar bhrí spioradálta éigin i bhfolach i nithe pléisiúrtha an tsaoil seo. Le linn na saoire, seastar siar ar feadh tréimhse as rithim agus as struchtúr na maireachtála gnáthlaethúla. Cuirtear cuid de na gnáthrialacha sin ar fionraí le linn an turais. Ceadaíonn an turasóir dó féin, b’fhéidir, é féin a iompar ar shlí nach ndéanfadh sé sa bhaile, saortha mar atá sé óna mbítear ag súil leis uaidh go hiondúil. Ar ndóigh, thig leis sin fadhbanna a chruthú. Má mhothaíonn an taistealaí Gaelach go bhfuil sé saor ar na gnáthrialacha agus na gnáthdhualgais a bhíonn air sa bhaile, ní hamhlaidh don dúchasach nach bhfuil ar saoire agus a bhfuil a chuid coinbhinsean cultúrtha féin aige, coinbhinsin atá níos 'coimeádaî' go minic ná cuid coinbhinsean an taistealaí.

\section{Sárú rialacha agus íogaireachtaí cultúrtha}

Bíonn an turasóir 'cultúrtha' in amanna ag iarraidh é féin a idirdhealú 'ó dhaoscarshlua na turasóireachta' (Somers 2002: 14) agus ón 'tsoibealtacht iartharach' (Ó Searcaigh 2004: 95), ón turasóir neamh-íogair sin a labhraíonn ró-ard, nach bhfuil i dtiúin puinn le cultúr na háite nó a bhrúnn a cheamara go míbhéasach i ngnéithe leochaileacha de shaol na ndúchasach, mar a dhéanann an bhean áirithe seo a leanas i Neipeal:

Fad is a bhí na deasghnátha créamtha seo ag gabháil ar aghaidh bhí boitseachán mór mná, Meiriceánach, gan fios gnoithe ar bith, ag glacadh grianghraf, gach 'Gosh!' aici le hiontas, a ceamara ag cliceáil fad is a bhí an corp á loscadh. Nuair a iarradh uirthi a ceamara a choinneáil ar shiúl ón charn créamtha chuala mé í ag rá os ard, 'Isn't this a public burning? Surely I can take snapshots. Back in the States my family would sure love to see all of this. Your culture is so photogenic.' Is beag nár léim mé as mo chraiceann le fearg nuair a chuala mé sin. Chuaigh mé anonn chuici agus dúirt mé léi gur mhór an náire di a leithéid d'ailp easurramach chainte a ligean as a béal ar an ócáid ghoilliúnach (Ó Searcaigh 2004: 23).

Mar sin féin, tugann an léitheoir faoi deara go bhfuil grianghraf de láthair chréamtha sa leabhar, rud a mheabhraíonn dúinn go mb'fhéidir go mbíonn a chaochspota féin ag an turasóir chultúrtha, dá íogaire. Ní hannamh fadhbanna sin na turasóireachta á bplé sa litríocht taistil. Dar le Rosenstock agus Magan beirt, ní fhanann an turasóir fada go leor in aon áit chun aithne a chur ar an mhaireachtáil ann; agus is ag treoirleabhair nó ag treoraithe a bhíonn sé á stiúradh, agus bíonn sé gearrtha amach ó réaltacht na ndúchasach; ní dhéanann sé fiú iarracht leis an teanga áitiúil:

Táimid ag iarraidh teacht isteach ar an teanga áitiúil in Kerala. Nandhi is ea 'gura maith agat' agus nale kannam is ea 'feicfidh mé amárach thú'. Tugaim faoi deara gur beag eachtrannach thart anseo a dhéanann aon iarracht in aon chor ar aon siolla eile a fhuaimniú ach Gearmáinis nó Béarla (Rosenstock 2003: 38).

Tar éis tiomáint lae, stopamar i Chefchaoen, baile Burba i measc Sléibhte Atlais. Agus an campa á chur suas againn bhí aoirí ag fánaíocht thart, súil amháin ar a gcaoirigh acu, an tsúil eile orainne. Chuir an íomhá iontas an domhain orm. Bhí cloiste go minic agam faoin saol simplí, sean-nósach a chleachtaítear i dtíortha neamhfhorbartha an domhain. Bhí sé feicthe agam i scannáin, léite agam faoi i leabhair agus cloiste agam i scéalta, agus anois, faoi dheireadh thiar thall, bhíos ar an láthair. B'é seo an phríomhchúis dom a bheith ar an dturas seo is dócha — chun cultúr eile a bhlaiseadh.

...(T)háinig imní mhór orm nuair a chonaiceas conas mar a bhí mo chompánaigh ag déileáil le muintir na háite a bhí ag éirí níos fiosraí le gach 
nóiméad a d'imigh thart. Ní raibh fiú na pubaill curtha suas nó an tine lasta againn nuair a tháinig na haoirí ag síneadh lámh na cairdiúlachta chugainn. Chuir sé brón millteach orm a fheiscint mar a dhiúltaíomar dóibh.

...(C)heapas gur cheart dúinn ar a laghad beannú dóibh.

...nach bhféadfaimis fiú cúpla focal a labhairt leo? Bhíos féin, ach go háirithe, ag tnúth le bualadh le muintir na háite, ach níor dheineamar faic. Dhiúltaíomar dóibh, agus is náir dom a rá, fiú le mo chuid smaointe ardnósacha, bhíos chomh doicheallach leis an gcuid eile acu. Níor dheineas fiú miongháire leo. Bhíos róthugtha lena bheith mar dhuine den ghrúpa.... Dhein duine nó beirt den ghrúpa fonóid faoina mBéarla briste, fiú. Chuir sé seo goimh orm toisc gur bhriste go mór mo chuid Fraincise seachas a gcuid Béarla siúd, agus ní raibh éinne ag fonóid fúmsa i rith an lae. Gan dabht ní raibh focal Fraincise ina bpluic ag an lucht fonóide a bhí inár measc-na (Magan 1998: 16-17).

\section{Dearcadh an turasóra}

Is eagal le turasóir íogair, ar nós Magan, go bhféadfadh grinndearcadh an taistealaí ar na dúchasaigh ábhar íontais nó órnáidí nó fiú feic shaolta a dhéanamh díobh. Aithníonn sé gur soiléire an chontúirt sin oibiachtú a dhéanamh ar na dúchasaigh nuair is ag taisteal le ceamara teilifíse a bhíonn an turasóir. Feileann an t-andúchas don teilifís, rud allta suimiúil a dtig linn breathnú air (gan é a bheith ábalta breathnú ar ais orainn). Tuigeann Manchán Magan an chontúirt sin agus clár teilifíse á dhéanamh aige san India. Ar an Ghainséis dó, leanann Magan leis an toise eiticiúil sin a phlé lena dheartháir agus le fear an bháid, ar Indiach é:

\section{D’fhiafraigh mé den bhádóir cad a cheap na Hiondúigh i dtaobh na dturasóirí a stánann orthu agus iad i mbun a gcuid níocháin agus paidreoireachta. 'Fáiltímid roimh gach éinne, den chuid is mó, ar seisean.}

'Is fearr go mór é ná laethanta an Raj, nuair nach raibh suim ar bith ag na Sasanaigh ionainn.'

Dúirt sé gurb é an créamadh an t-aon rud gur cheart ómós ar leith a thabhairt dó. Bhí fáilte roimh éinne faire air — ba dheasghnáth poiblí é — ach gan pictiúir a thógaint. Ó am go chéile, d'éiríodh an lucht caointe chomh feargach le turasóirí le ceamaraí go n-ionsaídís iad. Gortaíodh fear ón Eilbhéis go dona cúpla seachtain roimhe, agus cinnte, ní fhéadfaí a rá nár thuig sé cad a bhí á dhéanamh aige: bhí fógra i ngach óstán ag rá go raibh sé i gcoinne an dlí grianghraif a thógaint.

D'aontaigh an Tíogar gur cheart urraim ar leith a thabhairt do chleachtais báis gach cultúir, ach dúirt go raibh an scéal beagán difriúil nuair a bhí clár teilifíse á dhéanamh ag duine.... Phléamar an tuairim ar feadh tamaill, go dtí gur cuireadh deireadh tobann leis nuair a chonaiceamar radharc de chorpán faoi thaiséadach ag gluaiseacht thar an bhfuinneog ar rafta bambú.... Rug an Tíogar greim ar an gceamara agus rith ina dhiaidh, fad is a bhí mise ag íoc an bhille agus ag fágaint slán ag an mbádóir (Magan 2005: 96, 97).

Ceistíonn Ó Searcaigh dearcadh s'aige an turasóra chomh maith:

Tráthnóna i dTír Tairngire a bhí ann, gnaoi na gréine ar an duine agus ar an dúlra. Cha samhlófá go dtiocfadh le pian nó piolóid, bás nó bochtanas a bheith mar chuid de dhán an duine sa tsolas lách, soilíosach seo.... Chan den tsaol seo an scéimh seo, arsa mise liom féin, ach den tsíoraíocht. Ach bhí a fhios agam i mo chroí istigh nach raibh ansin ach rómánsaíocht bhaoth an turasóra. Bhí mé ag leagan barraíocht béime ar an tsúil agus gan leath go leor ar an stuaim (Ó Searcaigh, 2004: 114).

An ndéanann dearcadh an taistealaí éagóir ar an dúchasach bhocht? An mbriseann 'dearcadh' an cheamara isteach ar phríobháid agus ar dhínit an dúchasaigh? I gcasadh suimiúil, tuigtear do Magan ag pointe eile ina chuntas nach bhfuil aon difear i ndáiríre idir an t-éileamh eiticiúil a dhéanann a chomhthurasóir Eorpach air, gur ceist dhaonna, 
seachas ceist chultúrtha í; go bhfuil an éagóir chéanna á himirt aige sa dá chás. Tá dearcadh an cheamara á thiontú aige ar hipí mná, Muriel, bean atá anchorraithe inti féin agus ar a cairde (Magan 2005: 137-147). Tuigtear do Magan go bhfuil sé féin agus a cheamara teilifíse ag briseadh isteach ar phríobháid na mná sin agus a cuid cairde cuidsúlacha. Tuigtear dó go bhfuil sé, chan amháin, ag baint den dínit s'aici, ach fosta ag baint den dínit s'aige féin chomh maith. Tuigtear don léitheoir gur ar an mheán féin, an teilifís, atá an locht:

Gan dabht, nuair a chuala mé ón Tíogar go mbeadh sraith teilifise á déanamh againn, d'fhiafraigh mé de dhaoine cathain a bheadh an ghealach lán arís. Thuig mé go bhféachfadh an chóisir go hiontach ar an teilifís. Hippies ag cuimilt cailíní nochta le cleití ba ea díreach an rud a bhí ó theanga na Gaeilge....

Nuair a d'inis mé don Tíogar faoi Muriel ar an nguthán, d'impigh sé orm cuairt a thabhairt uirthi agus tathant uirthi agallamh a dhéanamh linn. Gan dabht, rinne mé rud air. Chuaigh mé suas go Kasa Devi le fáil amach cá raibh sí ina cónaí, agus dhírigh duine éigin i dtreo scioból tuí mé. Ba i gcúinne beag i lochta an sciobóil a tháinig mé uirthi. Bhí sí ag athrá mantra liriciúil casta gan stad, agus ag cniotáil ag an am céanna. Gan dabht, níor chuimhin léi mé, agus nuair a d'inis mé di céard a bhí uaim, chaith sí seile orm agus dúirt gur shuarachán saobh mé. An rud is measa ná, in ionad náire a bheith orm, bhí doicheall orm nár ghéill sí dom, nár lig sí dúinn scéal corraitheach teilifíse a dhéanamh di. Bhí gáifeachas agus mí-ionracas an mheáin ag dul i gcion orm cheana féin (Magan 2005: 149, 150).

Sa léamh a dhéanann Eimear Ní Chinnéide ar shaothar Magan chun an gaol idir an Gael óg agus pobal dúchasach na hIndia a phlé, go háirithe maidir le híogaireachtaí cultúrtha ar nós scannanú an chréamtha, tarraingíonn sí go mór ar théis Edward Said in Orientalism (1978). Maíonn Said go bhfuil an leagan amach atá ag Iartharaigh ar an chuid eile den domhan ciníoch, ordlathach agus bunaithe ar chumhacht agus ar fhorlámhas. Dar le Said, tá léiriú an Oirthir in ealaín an Iarthair, an litríocht taistil san áireamh, le tuiscint mar chuid de heigiméine an
Iarthair. Cé chomh hiontaofa is atá téis sin Said, ag scríobh mar atá sé ó pheirspictíocht an mharxachais chultúrtha? Dar le Robert Irwin, níl saothar sin Said, Orientalism iontaofa mar thaighde. Dar leis, tá léamh polaitiúil Said chomh claonta sa leabhar sin gur 'malignant charlatanry' atá ann (Irwin 2006: 5). Cé go luíonn plé níos cuimsithí ar leabhair sin Said agus Irwin taobh amuigh de raon na haiste seo, is fiú cuimhneamh nach gá do Mhanchán Magan, nó d'aon duine eile, a chuid taistil a chur i gcrích nó a thuiscint de réir théarmaí tagartha teoiricíochta éigin nó idé-eolaíochta éigin atá faiseanta i gcoda áirithe den acadamh anglafónach i láthair na huaire.

\section{'Oilithreacht' faoi m'anam}

Is bealach seoigh é an taisteal ag duine chun a shaol a thuiscint, chun spás a chur idir é féin agus gnáthshaol an bhaile. Bealach é chun mapáil éigin a dhéanamh ar chúrsa a shaoil. Is turas taighde agus inspioráide é an taisteal do na húdair seo i gcoitinne, ach, i gcás Magan de, is soiléire fós gur cuid dá phróiseas féintuisceana agus dá fhéinchur in iúl é an taisteal. Is 'oilithreacht faoina anam'é:

Cheap daoine áirithe aige baile go rabhas ag teitheadh ó ghlacadh le bás m’athar trí thaisteal. Dá mb'fhíor é, ní raibh sé ag déanamh aon mhaitheas dom. Bhí sé fós marbh, agus ba bheag seans go dtiocfainn ar aon mhíorúilt sa mhór-roinn a thabharfadh thar n-ais chun na beatha é (Magan 1998: 71).

...fuaireas cuntas iomlán ó mo dhlúthchara a bhí ag gabháil le haisteoireacht i mBristó i dtaobh a shaoil ag strácáil trí fhadhbanna pearsanta i measc aisteoirí so-chorraithe. D'airíos gurbh aisteach dom a fhéin-scrúdú iomarcach tar éis dom a bheith i measc nithiúlacht thíriúil an ghrúpa. Bhíos ag teacht ar thuiscint nach raibh gá le féinmhachnamh síoraí agus gur fearr glacadh le mórán den saol agus den phearsantacht gan cheistiú go dtí go bhfaighfí breis céille (Magan 1998: 114).

Cuireann an turas ar chumas an duine machnamh faoi threoir agus faoi bhrí a shaoil, gan amhras. Chan iontas go mbeadh an ghné sin níos láidre sna cuntais 
'oilithreachta'. Díol suime, sa chomhthéacs seo, tuairim Bauman go bhfuil an tuiscint thraidisiúnta den bheatha mar oilithreacht imithe ar gcúl san Iarthar, agus tuiscint eile tagtha chun cinn atá níos gaire d'eispéireas na turasóireachta (2011: 18-35). Dar le Bauman, is saintréith de chuid na maireachtála sa ré chomhaimseartha san Iarthar drogall a bheith ar dhaoine iad féin a cheangal go ródhlúth nó go buan le daoine eile: gaiste is ea an dlúthnasc, de réir dealraimh, agus bua is ea an tsolúbthacht, dá réir. In ainneoin na háibhéile atá i dtéis sin Bauman, is díol suime a thuairim gur de dhlúth-thaithí an duine chomhaimseartha ar a shaol féin a bheatha a bhrath mar eispéireas turasóireachta (seachas mar oilithreacht) (2011: 18-35). An t-eispéireas sin agus míshástacht leis sin, b'fhéidir, is cúis leis an ráchairt atá ar an tsiúlóid fhada go Santiago da Compostella, ar oilithreacht 1 atá i ndiaidh a hathbheochana ag lucht mhargaíocht na turasóireachta. Tá trí chuntas ó Ghaeil againn (de Barra 2007; Mac Brádaigh 2015; Ó Muirthile 2017). Le linn a shiúlóide fada oilithreachta a ndéantar cur síos air in An Bóthar go Santiago (2007) foilsítear léaspairtí féintuisceana do Mhícheál de Barra:

Is fearr a thuigim gur tabhartas ó Dhia an inbhreathnaitheacht atá ionam ó dhúchas. Shíl mé ar feadh na mblianta gur mallacht a bhí inti. Cuireann sí iachall orm tréimhsí a chaitheamh 'i mo dhíthreabhach' ar mo mharana san uaigneas ag sú isteach brí agus fuinneamh agus spreacadh glóir agus niamhracht Dé a bhíonn ag lonrú trí áilleacht an domhain nádurtha....

Lá agus mé ag tógáil sosa ag cuaille ciliméadair aistear lae ó Compostela, bhuail smaoineamh mé amhail buille casúir, go bhfuil an chuid is mó de mo shaol thart agus nach bhfuil mórán le taispeáint agam mar thoradh ar na blianta a bhronn Dia orm. Má tá maitheas ar bith déanta agam, is mionrudaí iad, ar nós píosa beag de bhóthar na beatha a shiúl le daoine óga in am an ghátair nó iarracht bheag a dhéanamh chun an scála a chothromú i gcás daltaí laga. An-chuid gníomhartha, éachtaí uaireanta, curtha i gcrích agam ach gan iad bunaithe ar an ngrá. Tuigim anois nach fiú tada iad.
Ar chúis éigin agus mé ag siúl de thruslóga fada trasna na Spáinne, bhíodh m’aigne ag gabháil siar, í ag spaisteoireacht go réagánta trí chuimhní cinn ó móige aníos. Casadh daoine orm ar bhóithrín úd na gcuimhní ar mhaith liom maithiúnas a iarraidh orthu. Chuir cuimhní ar dhaoine eile an fhearg ag brúchtaíl aníos ionam. Gan leigheas air anois ach maithiúnas a bhronnadh orm féin agus orthusan. An rud is mó a chuir iontas orm ná an líon mór daoine uaisle, cineálta, tacúla a choimeáad cuideachta liom ar stráicí de bhóthar na beatha....

Tháinig léargais phríobháideacha eile chugam nach luafaidh mé anseo agus cinn eile fós nach bhfuil a fhios agam iad a bheith ann, a déarfainn (de Barra 2007: 294-295).

Is cosúil go mbíonn toise quasi-meitifisiciúil nó quasispioradálta ag baint le tóraíocht phearsanta an taistil. Is cosúil go mbíonn fáil éigin ar rud éigin idir dhá thír nach bhfuil ar fáil chomh réidh sa bhaile. Bíogann an taisteal i gcéin an tsamhlaíocht chun dul isteach i limistéir nua, mar a dhéanann an litríocht mhaith. Tá téamaí áirithe antraipeolaíocha le haithint sa litríocht taistil, faoi mar a luadh san aiste: an tóir ar chaidreamh le strainséirí agus an tóir ar chineál eile caidrimh; an tóir ar 'aeistéitic' nó ar stíl phearsanta; an tóir ar thuiscint níos doimhne den bhaile; an tóir ar chastachtaí cultúrtha an tsaoil a thuiscint. Ach i dteannta na dtéamaí sin, níor mhiste, chun nach gcaillfí draíocht mheallacach agus pléisiúr an taistil, smaoineamh ar an turasóireacht mar shaothar de chuid na samhlaíochta, saothar a bhfuil rud éigin i bpáirt aige leis an litríocht chruthaitheach. Chuige sin, d'fhéach an aiste seo le coincheapa áirithe a mholadh (an t-eispéireas teorann, an dul le sruth, agus an carnabhalachas) a d'fhéadfaí a úsáid chun an taisteal agus an litríocht a éiríonn aisti araon a thuiscint ar shlí a thugann a cheart do ról na samhlaíochta agus don chruthaitheacht. Más suim linn an litríocht taistil mar litríocht, seachas mar cháipéis shóisialta a ndéanfaí léamh polaitiúil uirthi, ní mór tábhacht an phléisiúir sa taisteal a aithint agus modh eile léitheoireachta a fhorbairt, dá réir. 


\section{SAOTHAIR A CEADAÍODH}

Bahktin, M. (1984) Rabelais and his World. Bloomington: Indiana University Press.

Bauman, Z. (2011) 'From Pilgrim to Tourist, or a Short History of Identity'. In: Hall, S. and du Gay, P. Questions of Cultural Identity. London: Sage Publications, 18-36.

Csikszentmihayi, M. (1992) Flow: The Psychology of Happiness. London: Ryder and Co.

de Barra, M. (2007) An Bóthar go Santiago. Baile Átha Cliath: Cois Life.

Cronin, M. (2000) 'An Domhan trí mheán na Gaeilge' "Ag Taisteal dom 'mach”. In: Ó Cearúil, M. (eag.) Aimsir Óg 2000 Cuid a Dó. Baile Átha Cliath: Coiscéim: 289-295.

Harrison, J. (1993) Being a Tourist: Finding Meaning in Pleasure Travel. Vancouver: University of British Columbia Press.

Irwin, R. (2006) For Lust of Knowing: The Orientalists and their Enemies. London: Allen Lane / Penguin.

Mac Brádaigh, F. (2015) Conair na Fraince go Santiago. Baile Átha Cliath: FÁS.

Mac Liammóir, M. (1956) Aisteoirí Faoi Dhá Sholas. Baile Átha Cliath: Sáirséal agus Dill.

Magan, M. (1998) Manchán Ar Seachrán: Ó Bhaile Atha Cliath go Nairobi i Seanleorai Airm. Baile Átha Cliath: Coiscéim.

Magan, M. (2005) Baba-li agus TnaG: Seachrán san India. Baile Átha Cliath: Coiscéim.

Mac Síthigh, D. (2013) Iomramb Bhréanainn MMXII: Ón Daingean go dti an Ioslainn. An Daingean: An Sagart.

Ní Chinnéide, E. (2015) 'Teagmháil idirchultúrtha i Litríocht Taistil Mhancháin Magan'. In: Nic Congáil, R. et al. (eag.) Litriocht na Gaeilge ar fud an Dombain: Critic, Cultúr agus Combthéacs Iml. II. Baile Átha Cliath: LeabhairCOMHAR: 259-278.

Ní Dhonnchadha, A. agus Nic Eoin, M. (2008) Ar an gCoigrioch: Diolaim Litriochta ar Scéal na bImirce. Indreabhán: Cló Iar-Chonnachta.

Ní Mhaoileoin, Ú. (1958) Le Grá Ó Úna. Baile Átha Cliath: Sáirséal agus Dill.

Ní Mhaoileoin, Ú. (1967) An Maith Leat Spaigití. Baile Átha Cliath: Sáirséal agus Dill.

Ní Mhaoileoin, Ú. (1969) Turas go Túinis. Baile Átha Cliath: Sáirséal agus Dill.
Nic Giolla Bhríde, N. (2003) Mise agus na Maighigh. Baile Átha Cliath: Coiscéim.

Ó Cathasaigh, A. (1998) Caiscin: Altanna san Irish Times 1953/56 le Máirtin Ó Cadhain. Baile Átha Cliath: Coiscéim.

Ó Grianna, S. (1945) Saol Corrach. Baile Átha Cliath: An Press Náisiúnta.

Ó Muirthile, L. (2017) Oilithreach Pinn. Baile Átha Cliath: Cois Life.

Ó Rinn, L. (1931) Turus go Páras. Baile Átha Cliath: Oifig Díolta Foilseacháin Rialtais.

Ó Searcaigh, C. (2004) Seal i Neipeal. Indreabhán: Cló Iar-Chonnachta.

Reidy, F. (2009) Ó Chósta go Cósta. Indreabhán: Cló Iar-Chonnacht.

Rosenstock, G. (2003) Ólann mo Mbiúil as an nGainséis. Indreabhán: Cló Iar-Chonnachta.

Said, E. (1978) Orientalism. New York: Pantheon Books.

Somers, D. (2002) Rince ar na Ballaí. Baile Átha Cliath: Cois Life.

Taylor, C. (2007) A Secular Age. London: The Belknap Press of Harvard University.

Uí Chíobháin, K. (2015) 'Teanga agus Féiniúlacht Náisiúnta i Scríbhneoireacht Taistil na Gaeilge faoin Mór-Roinn, 1900-1973’. In: Nic Congáil, R. et al. (eag.) Litriocht na Gaeilge ar fud an Dombain: Critic, Cultúr agus Combthéacs Iml. II. Baile Átha Cliath: LeabhairCOMHAR: 243-258. 